\title{
Hamilton's Gradient Estimates and A Monotonicity Formula for Heat Flows on Metric Measure Spaces
}

\author{
Renjin Jiang \& Huichun Zhang
}

\begin{abstract}
In this paper, we extend the Hamilton's gradient estimates [16] and a monotonicity formula of entropy [25] for heat flows from smooth Riemannian manifolds to (non-smooth) metric measure spaces with appropriate Riemannian curvature-dimension condition.
\end{abstract}

\section{Introduction}

In their fundamental work [23], Li and Yau discovered a gradient estimate for positive solutions of the heat equation

$$
\partial_{t} u=\Delta u
$$

on a smooth Riemannian manifold with Ricci curvature bounded from below. Later on, Hamilton in [16] used the similar method to establish the following gradient estimates.

Theorem 1.1 (Hamiton [16]). Let $(M, g)$ be an n-dimensional compact Riemannian manifold with Ricci curvature $R_{i j} \geqslant-K g_{i j}$ for some $K \geqslant 0$ and $\partial M=\varnothing$. If $u(x, t)$ is a positive solution of the heat equation with $0<u \leqslant M$. then

$$
t|\nabla \log u|^{2} \leqslant(1+2 K t) \cdot \log (M / u)
$$

Such type gradient estimates are called as Li-Yau-Hamilton (LYH, for short) inequalities afterwards. Recently, Kotschwar [20] extended Theorem 1.1 to complete noncompact manifolds.

The LYH inequality is of the basic tools, that has been widely used in geometric analysis. For instance, the LYH inequalities imply the classical Harnack inequalities for heat equations, by integrating the LYH type gradient estimates along space-time curves. The research of LiYau-Hamilton estimates for heat equation (or other geometric evolution equations) on smooth Riemannian manifolds has a long history. For an overview, the reader is referred to Chapter 4 in book [31] and [27, 21, 37, 28, 7, 8], and references therein. Very recently, Bakry-Bolley-Gentil [7] have established an optimal global Li-Yau on smooth Markov semi-group under a curvaturedimension condition.

2010 Mathematics Subject Classification. 53C23, 35K05

Key words and phrases. Metric measure space, Curvature-dimension condition, Harmilton's gradient estimates, Entropy.

R.Jiang is partially supported by NSFC (No. 11301029); H.Zhang is partially supported by NSFC (No. 11201492) and the Fundamental Research Funds for the Central Universities. 
Li-Yau's gradient estimates have been extended from smooth manifolds to compact Alexandrov spaces in [29], and recently to general metric measure spaces with the Riemannian curvaturedimension condition $R C D^{*}(K, N)$, by Garofalo-Mondino [12] (for the case $\mu(X)<\infty$ ) and [19] (for the case $\mu(X)=\infty$ ). Gradient estimates for harmonic functions on metric measure spaces have been studied in [17, 18]. We refer the readers to [1, 2, 3, 5, 6, 11] for recent developments for Riemannian curvature dimension conditions $R C D^{*}(K, N)$, and [24, 34, 35] for curvature dimension conditions $C D(K, N)$, on metric measure spaces; see Section 2 below.

One of our main aim of this paper is to consider the LYH inequality for heat equations on non-smooth metric measure spaces $(X, d, \mu)$. Precisely, our first main result is the following:

Theorem 1.2. Let $(X, d, \mu)$ be a proper metric measure space satisfying $R C D^{*}(K, \infty)$, where $K \leqslant$ 0 . Let $u(x, t)$ be a positive solution of the heat equation on $X \times[0, \infty)$ with initial value $u(x, 0)=$ $u_{0}(x) \in \cup_{1 \leqslant q<\infty} L^{q}(X)$. Suppose that there exists a positive constant $M$ such that $u_{0}(x) \leqslant M$ for almost every $x \in X$. Then

$$
t|\nabla \log u|^{2} \leqslant(1-2 K t) \cdot \log (M / u)
$$

for almost every $(x, t)$ in $X \times[0, \infty)$.

On the other hand, inspired by Perelman's $\mathcal{W}$-entropy, Ni [25] introduced an entropy for the heat equation on an $n$-dimensional smooth Riemannian manifold $(M, g)$. For any smooth function $f$ on $M$ and $\tau>0$ with $\int_{M}(4 \pi \tau)^{-n / 2} e^{-f} d x=1$, the entropy is given as

$$
\mathcal{W}(f, \tau):=\int_{M}\left(\tau|\nabla f|^{2}+f-n\right) \frac{e^{-f}}{(4 \pi \tau)^{n / 2}} d x .
$$

Let $u(x, t)$ be a positive solution of (1.1) on a closed (i.e., compact and without boundary) manifold $M$ with $\int_{M} u d x=1$. Let $f$ be defined as $u=(4 \pi \tau)^{-n / 2} e^{-f}$ and $\tau=\tau(t)$ with $\frac{d \tau}{d t}=1$. In [25], Ni proved that, if $M$ has nonnegative Ricci curvature, then the entropy $\mathcal{W}(f, \tau)$ is monotone decreasing along the heat equation (as $t \rightarrow \infty$ ). Such monotonicity also discussed in [10]. Wang [36] extended it to a compact manifold with Bakry-Émery curvature bounded below.

Our second main result is to extend Ni's monotonicity for heat equation to non-smooth metric measure space $(X, d, \mu)$. Precisely, we have the following result.

Theorem 1.3. Let $(X, d, \mu)$ be a compact metric measure space satisfying $R C D^{*}(0, N)$ with $N \in$ $[1, \infty]$. Let $u(x, t)$ be a positive solution of the heat equation on $X \times[0, \infty)$ with initial value $u(x, 0)=u_{0}(x) \in L^{\infty}(X)$. Then we have the following:

(i) If $N=\infty$, by letting $f:=-\log u$, then the entropy

$$
\mathcal{W}_{\infty}(f, t):=\int_{X}|\nabla f|^{2} \cdot e^{-f} d \mu
$$

is monotone decreasing (as $t \rightarrow \infty$ ).

(ii) If $N<\infty$, by letting $f$ be defined as $u=(4 \pi \tau)^{-N / 2} e^{-f}$ and $\tau=\tau(t)>0$ with $\frac{d \tau}{d t}=1$, then the entropy

$$
\mathcal{W}_{N}(f, \tau):=\int_{M}\left(\tau|\nabla f|^{2}+f-N\right) \frac{e^{-f}}{(4 \pi \tau)^{N / 2}} d \mu
$$

is monotone decreasing (as $t \rightarrow \infty$ ). 
According to the referee's suggestion, we post the following question:

Question 1.1. Extend Theorem 1.3 to the case when $(X, d, \mu)$ is not compact and $\mu$ is a $\sigma$-finite measure on $X$.

The paper is organized as follows. In Section 2, we recall some necessary materials for Sobolev spaces and Riemannian curvature-dimension condition on metric measure spaces. Hamilton's gradient estimate (1.2) will be proved in the third section. In the last section, we will prove the monotonicity result Theorem 1.3

Acknowledgments. We are grateful to the anonymous referee for his/her several interesting and helpful suggestions and comments, which improves this paper.

\section{Preliminaries}

In this section, we recall some basic notions and several auxiliary results. Let $(X, d)$ be a complete, separable and proper metric space, $\mu$ be a $\sigma$-finite Radon measure, with $\operatorname{supp}(\mu)=X$.

\subsection{Sobolev spaces on metric measure spaces}

Let $C([0,1], X)$ be the space of continuous curves on $[0,1]$ with values in $X$, which we endow with the sup norm. For $t \in[0,1]$, the map $e_{t}: C([0,1], X) \rightarrow X$ is the evaluation at time $t$ defined by

$$
e_{t}(\gamma):=\gamma_{t}
$$

Given a non-trivial closed interval $I \subset \mathbb{R}$, a curve $\gamma: I \rightarrow X$ is in the absolutely continuous class $A C^{q}([0,1], X)$ for some $q \in[1, \infty]$, if there exists $f \in L^{q}(I)$ such that, for all $s, t \in I$ and $s<t$, it holds

$$
d\left(\gamma_{t}, \gamma_{s}\right) \leq \int_{s}^{t} f(r) d r
$$

Definition 2.1 (Test Plan). Let $\pi \in \mathcal{P}(C([0,1], X))$. We say that $\pi$ is a test plan if there exists $C>0$ such that

$$
\left(e_{t}\right)_{\sharp} \pi \leq C \mu, \forall t \in[0,1],
$$

and

$$
\iint_{0}^{1}\left|\dot{\gamma}_{t}\right|^{2} d t d \pi(\gamma)<\infty
$$

Definition 2.2 (Sobolev Space). The Sobolev class $S^{2}(X)$ (resp. $\left.S_{\text {loc }}^{2}(X)\right)$ is the space of all Borel functions $f: X \rightarrow \mathbb{R}$, for which there exists a non-negative function $G \in L^{2}(X)\left(\right.$ resp. $G \in L_{\text {loc }}^{2}(X)$ ) such that, for each test plan $\pi$, it holds

$$
\int\left|f\left(\gamma_{1}\right)-f\left(\gamma_{0}\right)\right| d \pi(\gamma) \leq \iint_{0}^{1} G\left(\gamma_{t}\right)\left|\dot{\gamma}_{t}\right|^{2} d t d \pi(\gamma)
$$


It has been proved that (see [9, 32, 4]), for each $f \in S^{2}(X)$, there exists a unique minimal $G$ in the $\mu$-a.e. sense such that (2.1) holds. We then denote the minimal $G$ by $|\nabla f|$ and call it the minimal weak upper gradient following [4].

We then define the in-homogeneous Sobolev space $W^{1,2}(X)$ as $S^{2}(X) \cap L^{2}(X)$ equipped with the norm

$$
\|f\|_{W^{1,2}(X)}:=\left(\|f\|_{L^{2}}^{2}+\|\| \nabla f \|_{L^{2}}^{2}\right)^{1 / 2} .
$$

Definition 2.3 (Local Sobolev Space). Let $\Omega \subset X$ be an open set. A Borel function $f: \Omega \rightarrow \mathbb{R}$ belongs to $S_{\mathrm{loc}}^{2}(\Omega)$, provided, for any Lipschitz function $\chi: X \rightarrow \mathbb{R}$ with $\operatorname{supp}(\chi) \subset \Omega$, it holds $f \chi \in S_{\mathrm{loc}}^{2}(X)$. In this case, the function $|\nabla f|: \Omega \rightarrow[0, \infty]$ is $\mu$-a.e. defined by

$$
|\nabla f|:=|\nabla(\chi f)|, \quad \mu-\text { a.e. on }\{\chi=1\},
$$

for any $\chi$ as above. The space $S^{2}(\Omega)$ is the collection of such $f$ with $|\nabla f| \in L^{2}(\Omega)$.

Notice that, if $\mu$ is locally doubling and $(X, d, \mu)$ supports a local weak $L^{2}$-Poincaré inequality, the Sobolev space $W^{1,2}(X)$ coincides with the Sobolev spaces based on upper gradients introduced by Cheeger [9] and Shanmugalingam [32]; see Ambrosio, Gigli and Savaré [4].

Fixed any open set $\Omega \subset X$. We denote by $\operatorname{Lip}(\Omega)$ (or $\operatorname{Lip}_{\text {loc }}(\Omega)$, or $\operatorname{Lip}_{0}(\Omega)$ ) the space of Lipschitz continuous functions on $\Omega$ (resp. or locally Lipschitz continuous functions, or Lipschitz continuous functions with compact support on $\Omega$ ). The local Sobolev space $W_{\text {loc }}^{1,2}(\Omega):=L_{\text {loc }}^{2}(\Omega) \cap$ $S_{\text {loc }}^{2}(\Omega)$, and the Sobolev space with compact support $W_{0}^{1,2}(\Omega)$ is defined as the completion of $\operatorname{Lip}_{0}(\Omega)$ with respect to the $W^{1,2}$-norm. We also denote $C_{0}(\Omega)$ by the continuous function with compact support on $\Omega$ with uniform norm.

\subsection{Differential structure and the Laplacian}

The following terminologies and results are mainly taken from [3, 13].

Definition 2.4 (Infinitesimally Hilbertian Space). Let $(X, d, \mu)$ be a metric measure space. We say that it is infinitesimally Hilbertian, provided $W^{1,2}(X)$ is a Hilbert space.

Notice that, from the definition, it follows that $(X, d, \mu)$ is infinitesimally Hilbertian if and only if, for any $f, g \in S^{2}(X)$, it holds

$$
\|\nabla(f+g)\|_{L^{2}(X)}^{2}+\|\| \nabla(f-g) \|_{L^{2}(X)}^{2}=2\left(\|\| \nabla f\left\|_{L^{2}(X)}^{2}+\right\| \nabla g \|_{L^{2}(X)}^{2}\right) .
$$

Definition 2.5. Let $(X, d, \mu)$ be an infinitesimally Hilbertian space, $\Omega \subset X$ an open set and $f, g \in$ $S_{\mathrm{loc}}^{2}(\Omega)$. The map $\langle\nabla f, \nabla g\rangle: \Omega \rightarrow \mathbb{R}$ is $\mu$-a.e. defined as

$$
\langle\nabla f, \nabla g\rangle:=\inf _{\epsilon>0} \frac{|\nabla(g+\epsilon f)|^{2}-|\nabla g|^{2}}{2 \epsilon}
$$

the infimum being intended as $\mu$-essential infimum.

The inner product $\langle\nabla f, \nabla g\rangle$ is linear, and satisfies Cauchy-Schwartz inequality, Chain rule and Leibniz rule; see Gigli [13]. 
The inner product also provides a canonical strongly local Dirichlet form $\left(\mathscr{E}, W^{1,2}(X)\right)$ by

$$
\mathscr{E}(f, g):=\int_{X}\langle\nabla f, \nabla g\rangle d \mu, \quad \forall f, g \in W^{1,2}(X) .
$$

We denote by $\Delta_{\mathscr{E}}$ and $H_{t}$ the generator of $\mathscr{E}$ and the corresponding semigroup (heat flow) $e^{t \Delta_{\mathscr{E}}}$. The domain of the generator is denoted by $\mathscr{D}\left(\Delta_{\mathscr{E}}\right)$. For each $u_{0} \in L^{2}(X)$, the heat flow $H_{t} u_{0}(x)$ provides a (unique) solution of heat equation $\partial_{t} u=\Delta_{\mathscr{E}} u$ in $W^{1,2}(X)$.

The generator $\Delta_{\mathscr{E}}$ gives a natural definition of Laplacian. However, with the aid of the inner product, Gigli in [13] and Gigli-Mondino in [14] have defined a measure-valued Laplacian operator (or more general elliptic operators) as below (see also [26, 38] for the case of Alexandrov spaces).

Definition 2.6 (Laplacian). Let $(X, d, \mu)$ be an infinitesimally Hilbertian space. Let $f \in W_{\mathrm{loc}}^{1,2}(X)$. We call $f \in \mathscr{D}\left(\Delta^{*}\right)$, if there exists a signed Radon measure $v$ such that, for each $\psi \in \operatorname{Lip}_{0}(X)$, it holds

$$
-\int_{X}\langle\nabla f, \nabla \psi\rangle d \mu=\int_{X} \psi d v
$$

If such $v$ exists, it must be unique. We will write $\Delta^{*} f=v$.

We also denote by

$$
\mathscr{D}(\Delta):=\left\{f \in \mathscr{D}\left(\Delta^{*}\right): \Delta^{*} f \ll \mu \text { and the density } \frac{d\left(\Delta^{*} f\right)}{d \mu} \in L_{\mathrm{loc}}^{2}(X)\right\}
$$

When $f \in \mathscr{D}(\Delta)$, we denote $\Delta f:=\frac{d\left(\Delta^{*} f\right)}{d \mu}$.

Note that, if $f \in \mathscr{D}\left(\Delta^{*}\right)$, the test function $\psi$ can be chosen in $W_{0}^{1,2}(X) \cap L_{\mathrm{loc}}^{1}(X,|v|)$. The operator $\Delta^{*}$ is linear due to $(X, d, \mu)$ being infinitesimally Hilbertian.

It is clear that $\mathscr{D}\left(\Delta_{\mathscr{E}}\right) \subset \mathscr{D}(\Delta)\left(\subsetneq \mathscr{D}\left(\Delta^{*}\right)\right)$. Since we consider only that $X$ is proper, it is proved in [13, Proposition 4.24] that

$$
\mathscr{D}(\Delta) \cap W^{1,2}(X)=\mathscr{D}\left(\Delta_{\mathscr{E}}\right) \quad \text { and } \quad \Delta f=\Delta_{\mathscr{E}} f \quad \forall f \in \mathscr{D}\left(\Delta_{\mathscr{E}}\right) .
$$

Such a measure-valued Laplacian $\Delta^{*}$ satisfies the following Chain rule and Leibniz rule (we consider only the case where $X$ is proper):

Lemma 2.1. Let $(X, d, \mu)$ be infinitesimally Hilbertian and proper.

(i) (Chain rule) Let $g \in \mathscr{D}\left(\Delta^{*}\right) \cap L_{\mathrm{loc}}^{\infty}(X)$ and $\phi \in C_{\mathrm{loc}}^{1,1}(\mathbb{R})$. If $g \in C(X)$ or $g \in \mathscr{D}(\Delta)$, then we have

$$
\phi \circ g \in \mathscr{D}\left(\Delta^{*}\right) \quad \text { and } \quad \Delta^{*}(\phi \circ g)=\phi^{\prime} \circ g \cdot \Delta^{*} g+\phi^{\prime \prime} \circ g|\nabla g|^{2} \cdot \mu .
$$

(ii) (Leibniz rule) Let $g_{1}, g_{2} \in \mathscr{D}\left(\Delta^{*}\right)$. Then we have

$$
g_{1} \cdot g_{2} \in \mathscr{D}\left(\Delta^{*}\right) \quad \text { and } \quad \Delta^{*}\left(g_{1} \cdot g_{2}\right)=g_{1} \cdot \Delta^{*} g_{2}+g_{2} \cdot \Delta^{*} g_{1}+2\left\langle\nabla g_{1}, \nabla g_{2}\right\rangle \cdot \mu
$$

provided that $g$ satisfies one of the following three conditions:

(a) $g_{1}, g_{2} \in C(X)$;

(b) $g_{1}, g_{2} \in \mathscr{D}(\Delta) \cap L_{\mathrm{loc}}^{\infty}(X)$;

(c) $g_{2} \in W^{1,2}(X)$ and $g_{1} \in \operatorname{Lip}_{\text {loc }}(X) \cap \mathscr{D}(\Delta)$. 
Proof. Chain rule (i) and (a), (b) in Leibniz rule (ii) have proved in [13, Proposition 4.28 and Proposition 4.29] by Gigli. We need only to check the condition (c) in Leibniz rule.

We argue by approximation. Since $g_{2} \in W^{1,2}(X)$, it is shown in [3] that there exists a sequence $\left\{\widetilde{h}_{j}\right\}_{j=1}^{\infty} \subset \operatorname{Lip}(X) \cap W^{1,2}(X)$ such that

$$
\widetilde{h}_{j} \stackrel{L^{2}}{\rightarrow} g_{2} \quad \text { and } \quad\left|\nabla \widetilde{h}_{j}\right| \stackrel{L^{2}}{\rightarrow}\left|\nabla g_{2}\right|
$$

as $j \rightarrow \infty$. Notice that $W^{1,2}(X)$ is a Hilbert space (hence it is reflexive), we can use Mazur's lemma to conclude that there exists a convex combination of $\left\{\widetilde{h}_{j}\right\}$, denoted by $\left\{h_{j}\right\}$, which strongly converges to $g_{2}$ in $W^{1,2}(X)$. On the other hand, $\mathscr{D}\left(\Delta_{\mathscr{E}}\right)$ is also dense in $W^{1,2}(X)$. Thus, without loss of generality, we can assume that $h_{j} \in \operatorname{Lip}(X) \cap \mathscr{D}\left(\Delta_{\mathscr{E}}\right)$ for all $j$, and $h_{j} \stackrel{W^{1,2}(X)}{\rightarrow} g_{2}$.

Since $g_{1} \in \operatorname{Lip}_{\text {loc }}(X) \cap \mathscr{D}(\Delta)$ and $h_{j} \in \operatorname{Lip}(X) \cap \mathscr{D}\left(\Delta_{\mathscr{E}}\right)$, by (b), we have, for each $j \in \mathbb{N}$,

$$
\Delta^{*}\left(g_{1} \cdot h_{j}\right)=g_{1} \cdot \Delta^{*} h_{j}+h_{j} \cdot \Delta^{*} g_{1}+2\left\langle\nabla g_{1}, \nabla h_{j}\right\rangle \cdot \mu .
$$

Let us fix a test function $\phi \in \operatorname{Lip}_{0}(X)$. We have

$$
-\int_{X}\left\langle\nabla \phi, \nabla\left(g_{1} h_{j}\right)\right\rangle d \mu=-\int_{X}\left\langle\nabla\left(\phi g_{1}\right), \nabla h_{j}\right\rangle d \mu-\int_{X}\left\langle\nabla\left(h_{j} \phi\right), \nabla g_{1}\right\rangle d \mu+2 \int_{X} \phi \cdot\left\langle\nabla g_{1}, \nabla h_{j}\right\rangle d \mu .
$$

Notice that the combination of $g_{1} \in \operatorname{Lip}_{\mathrm{loc}}(X)$ and $h_{j} \stackrel{W^{1,2}(X)}{\rightarrow} g_{2}$ implies that $g_{1} \cdot h_{j} \stackrel{W_{\mathrm{loc}}^{1,2}(X)}{\rightarrow} g_{1} \cdot g_{2}$. The same holds for the sequence $\left\{\phi \cdot h_{j}\right\}$. Letting $j \rightarrow \infty$, we can get

$$
-\int_{X}\left\langle\nabla \phi, \nabla\left(g_{1} g_{2}\right)\right\rangle d \mu=-\int_{X}\left\langle\nabla\left(\phi g_{1}\right), \nabla g_{2}\right\rangle d \mu-\int_{X}\left\langle\nabla\left(\phi g_{2}\right), \nabla g_{1}\right\rangle d \mu+2 \int_{X} \phi \cdot\left\langle\nabla g_{1}, \nabla g_{2}\right\rangle d \mu .
$$

This proves the Lemma.

\subsection{Curvature-dimension conditions and consequences}

We shall use the following definition for $R C D^{*}(K, N)$ spaces, which is equivalent to the original definition [11, 6]. Here and in the sequel, for $K=0, \frac{2 K t}{e^{2 K t}-1}:=\lim _{K \rightarrow 0} \frac{2 K t}{e^{2 K t}-1}=1$.

Definition $2.7\left(R C D^{*}(K, N)\right.$ Space). Let $(X, d, \mu)$ be an infinitesimally Hilbertian space, and let $H_{t}$ be the semigroup corresponding to the previous Dirichlet form $\left(\mathscr{E}, W^{1,2}(X)\right)$.

Given $K \in \mathbb{R}$ and $N \in[1, \infty]$, the space $(X, d, \mu)$ is called a $R C D^{*}(K, N)$ space, if the following three conditions are satisfied:

(i) there exist $x_{0} \in X$, and constants $c_{1}, c_{2}>0$, such that

$$
\mu\left(B\left(x_{0}, r\right)\right) \leq c_{1} \cdot e^{c_{2} r^{2}}
$$

(ii) for all $f \in W^{1,2}(X)$ and each $t>0$, it holds for $\mu$-a.e. $x \in X$ that

$$
\left|\nabla H_{t} f(x)\right|^{2} \leq e^{-2 K t} H_{t}\left(|\nabla f|^{2}\right)(x)-\frac{4 K t^{2}}{N\left(e^{2 K t}-1\right)}\left|\Delta H_{t} f(x)\right|^{2},
$$

where, if $N=\infty$, the last term is understood as 0 ;

(iii) if $f \in W^{1,2}(X) \cap L^{\infty}(X)$ satisfying $|\nabla f| \leqslant 1$, then $f$ has an 1-Lipschitz representative. 
Let $(X, d, \mu)$ be a $R C D^{*}(K, N)$ space with $K \in \mathbb{R}$ and $N \in[1, \infty)$. Then the measure $\mu$ is local doubling, and hence $X$ is proper.

It is known that, (see [13, §4]), for each $t>0$, the operator $H_{t}$ is bounded from $L^{p}(X)$ to $L^{p}(X)$ for any $p \in[1, \infty)$. Thus, the semigroup (heat flow) can be extended on $L^{p}(X)$ for any $p \in[1, \infty)$. Therefore, for each $p \in[1, \infty)$ and $u_{0}(x) \in L^{p}(X)$, the heat flow $H_{t} u_{0}(x)$ provides a solution of heat equation $\partial_{t} u=\Delta u$. When $p \in(1, \infty)$, the $L^{p}$ solution of the heat equation is uniquely determined by its initial value in $L^{p}(X)$ (see [33, 22]).

Lemma $2.2\left([1,[2])\right.$. Let $K \in \mathbb{R}$, and let $(X, d, \mu)$ be a proper $R C D^{*}(K, \infty)$ space. Then, for any $t>0$ and $f \in L^{\infty}(X) \cap L^{2}(X)$, we have $H_{t} f \in \operatorname{Lip}(X)$ and

$$
\operatorname{Lip}\left(H_{t} f\right) \leqslant \frac{\|f\|_{L^{\infty}}}{\sqrt{2 \int_{0}^{t} e^{2 K s} d s}} .
$$

Our proof of Theorem 1.2 and Theorem[1.3 relies on some self-improvements of regularity for heat flows under the Riemannian curvature-dimension condition.

Lemma 2.3 ([30]). Let $K \in \mathbb{R}$ and let $(X, d, \mu)$ be a proper $R C D^{*}(K, \infty)$ space. If $f \in \mathscr{D}\left(\Delta_{\mathscr{E}}\right) \cap$ $\operatorname{Lip}(X) \cap L^{\infty}(X)$ with $\Delta f \in W^{1,2}(X)$. Then $|\nabla f|^{2} \in W^{1,2}(X) \cap L^{\infty}(X)$ and $|\nabla f|^{2} \in \mathscr{D}\left(\Delta^{*}\right)$. When we write the Lebesgue's decomposition of $\Delta^{*}\left(|\nabla f|^{2}\right)$ w.r.t $\mu$ as

$$
\Delta^{*}\left(|\nabla f|^{2}\right)=\Delta^{R}\left(|\nabla f|^{2}\right) \cdot \mu+\Delta^{S}(|\nabla f|)^{2},
$$

then we have the estimates that $\Delta^{S}(|\nabla f|)^{2} \geqslant 0$ and, for $\mu$-a.e. $x \in X$,

$$
\frac{1}{2} \Delta^{R}\left(|\nabla f|^{2}\right) \geqslant \frac{1}{N}(\Delta f)^{2}+\langle\nabla \Delta f, \nabla f\rangle+K|\nabla f|^{2} .
$$

Furthermore, we have, for $\mu$-a.e. $x \in\{y:|\nabla f(y)| \neq 0\}$,

$$
\frac{1}{2} \Delta^{R}\left(|\nabla f|^{2}\right) \geqslant \frac{1}{N}(\Delta f)^{2}+\langle\nabla \Delta f, \nabla f\rangle+K|\nabla f|^{2}+\frac{N}{N-1} \cdot\left(\frac{\left\langle\nabla f, \nabla|\nabla f|^{2}\right\rangle}{2|\nabla f|^{2}}-\frac{\Delta f}{N}\right)^{2} .
$$

Here and in the sequel, if $N=\infty$, then $\frac{1}{N}=0$ and $\frac{N}{N-1}=1$.

Proof. In the case of $N=\infty$, the lemma is Theorem 3.4 in [30].

In the case of $N<\infty$, the same argument in the proof of Lemma 3.2 in [30] implies (2.4) (See also Theorem 2.7 of [12]). The last inequality (2.5) can be proved by a modification of the argument in the proof of Theorem 3.4 in [30]. For completeness, we present a detailed proof as follows.

Fixed any three functions $f^{1}, f^{2}, f^{3}$ in

$$
\mathbb{D}_{\infty}:=\left\{f \in \mathscr{D}\left(\Delta_{\mathscr{E}}\right) \cap \operatorname{Lip}(X) \cap L^{\infty}(X) \mid \Delta f \in W^{1,2}(X)\right\},
$$

we choose the polynomial $\Phi: \mathbb{R}^{3} \rightarrow \mathbb{R}$ defined by (the same in [30])

$$
\Phi(\mathbf{f}):=\lambda f^{1}+\left(f^{2}-a\right)\left(f^{3}-b\right)-a b, \quad \lambda, a, b \in \mathbb{R},
$$


where $\mathbf{f}=\left(f^{1}, f^{2}, f^{3}\right)$. Now we have $\Phi(\mathbf{f}) \in \mathbb{D}_{\infty}$ by [30, Lemma 3.2], and

$$
\gamma_{2}(\Phi(\mathbf{f})) \geqslant K|\nabla \Phi(\mathbf{f})|^{2}+v \cdot(\Delta \Phi(\mathbf{f}))^{2}, \quad \mu-\text { a.e. in } X,
$$

where $v:=1 / N$, and $\gamma_{2}(f):=\frac{1}{2} \Delta^{R}\left(|\nabla f|^{2}\right)-\langle\nabla f, \nabla \Delta f\rangle$. According to [30], we know that for $\mu$-almost every $x \in X$ the inequality holds for every $(\lambda, a, b) \in \mathbb{R}^{3}$. Up to a $\mu$-negligible set, for every $x \in X$, we can take $a:=f^{2}(x)$ and $b:=f^{3}(x)$. Thus, we obtain

$$
\begin{aligned}
& \lambda^{2} \cdot \gamma_{2}\left(f^{1}\right)+4 \lambda \cdot H\left[f^{1}\right]\left(f^{2}, f^{3}\right)+2\left(\left|\nabla f^{2}\right|^{2} \cdot\left|\nabla f^{3}\right|^{2}+\left\langle\nabla f^{2}, \nabla f^{3}\right\rangle^{2}\right) \\
& \quad \geqslant K \lambda^{2} \cdot\left|\nabla f^{1}\right|^{2}+v \cdot\left(\lambda \cdot \Delta f^{1}+2\left\langle\nabla f^{2}, \nabla f^{3}\right\rangle\right)^{2} \\
& \quad=K \lambda^{2} \cdot\left|\nabla f^{1}\right|^{2}+v \cdot\left(\lambda^{2} \cdot\left(\Delta f^{1}\right)^{2}+4 \cdot \lambda \cdot \Delta f^{1}\left\langle\nabla f^{2}, \nabla f^{3}\right\rangle+4 \cdot\left\langle\nabla f^{2}, \nabla f^{3}\right\rangle^{2}\right),
\end{aligned}
$$

where $H[f](g, h):=\frac{1}{2} \cdot[\langle\nabla g, \nabla\langle\nabla f, \nabla h\rangle\rangle+\langle\nabla h, \nabla\langle\nabla f, \nabla g\rangle\rangle-\langle\nabla f, \nabla\langle\nabla g, \nabla h\rangle\rangle]$. We can rewrite inequality (2.6) as in the following form

$$
\begin{aligned}
0 \leqslant & \lambda^{2} \cdot\left(\gamma_{2}\left(f^{1}\right)-K \cdot\left|\nabla f^{1}\right|^{2}-v \cdot\left(\Delta f^{1}\right)^{2}\right)+\lambda \cdot\left(4 H\left[f^{1}\right]\left(f^{2}, f^{3}\right)-4 v \cdot \Delta f^{1}\left\langle\nabla f^{2}, \nabla f^{3}\right\rangle\right) \\
& +2\left(\left|\nabla f^{2}\right|^{2} \cdot\left|\nabla f^{3}\right|^{2}+\left\langle\nabla f^{2}, \nabla f^{3}\right\rangle^{2}\right)-4 v \cdot\left\langle\nabla f^{2}, \nabla f^{3}\right\rangle^{2} \\
:= & \mathcal{A} \cdot \lambda^{2}+\mathcal{B} \cdot \lambda+C .
\end{aligned}
$$

Since $\lambda$ is arbitrary, the coefficients $\mathcal{A}, \mathcal{B}$ and $C$ satisfy $\mathcal{B}^{2} \leqslant 4 \mathcal{A C}$. I.e.,

$$
\begin{aligned}
& 16 \cdot\left(H\left[f^{1}\right]\left(f^{2}, f^{3}\right)-v \cdot \Delta f^{1}\left\langle\nabla f^{2}, \nabla f^{3}\right\rangle\right)^{2} \\
& \quad \leqslant 4\left(\gamma_{2}\left(f^{1}\right)-K\left|\nabla f^{1}\right|^{2}-v\left(\Delta f^{1}\right)^{2}\right) \cdot\left[2\left(\left|\nabla f^{2}\right|^{2} \cdot\left|\nabla f^{3}\right|^{2}+\left\langle\nabla f^{2}, \nabla f^{3}\right\rangle^{2}\right)-4 v \cdot\left\langle\nabla f^{2}, \nabla f^{3}\right\rangle^{2}\right] .
\end{aligned}
$$

By using

$$
\left|\nabla f^{2}\right|^{2} \cdot\left|\nabla f^{3}\right|^{2}+\left\langle\nabla f^{2}, \nabla f^{3}\right\rangle^{2} \leqslant 2 \cdot\left|\nabla f^{2}\right|^{2} \cdot\left|\nabla f^{3}\right|^{2}
$$

and noting that $\mathcal{A} \geqslant 0$ (from (2.4)), the inequality 2.7) implies that

$$
\begin{aligned}
& \left(H\left[f^{1}\right]\left(f^{2}, f^{3}\right)-v \cdot \Delta f^{1}\left\langle\nabla f^{2}, \nabla f^{3}\right\rangle\right)^{2} \\
& \quad \leqslant\left(\gamma_{2}\left(f^{1}\right)-K\left|\nabla f^{1}\right|^{2}-v\left(\Delta f^{1}\right)^{2}\right) \cdot\left[\left|\nabla f^{2}\right|^{2} \cdot\left|\nabla f^{3}\right|^{2}-v \cdot\left\langle\nabla f^{2}, \nabla f^{3}\right\rangle^{2}\right] .
\end{aligned}
$$

Now we take $f^{1}=f^{2}=f^{3}=f$ and conclude that

$$
\left(\frac{1}{2}\left\langle\nabla f, \nabla|\nabla f|^{2}\right\rangle-v \cdot \Delta f \cdot|\nabla f|^{2}\right)^{2} \leqslant(1-v) \cdot|\nabla f|^{4} \cdot\left(\gamma_{2}(f)-K|\nabla f|^{2}-v(\Delta f)^{2}\right) .
$$

This implies (2.5) where $|\nabla f| \neq 0$. The proof is complete.

Remark 2.1. If $N=\infty$, we have $1 / N=0$ and $N /(N-1)=1$, and that the inequality (2.5) is

$$
\frac{1}{2} \Delta^{R}\left(|\nabla f|^{2}\right) \geqslant\langle\nabla \Delta f, \nabla f\rangle+K|\nabla f|^{2}+\left(\frac{\left\langle\nabla f, \nabla|\nabla f|^{2}\right\rangle}{2|\nabla f|^{2}}\right)^{2} .
$$


It is proved in [30] that

$$
\frac{1}{2} \Delta^{R}\left(|\nabla f|^{2}\right) \geqslant\langle\nabla \Delta f, \nabla f\rangle+K|\nabla f|^{2}+\frac{\left.\left.|\nabla| \nabla f\right|^{2}\right|^{2}}{4 \cdot|\nabla f|^{2}}
$$

This is stronger than (2.9), since $\left|\left\langle\nabla f, \nabla|\nabla f|^{2}\right\rangle\right| \leqslant\left.|\nabla f| \cdot|\nabla| \nabla f\right|^{2} \mid$.

\section{Hamilton's gradient estimates}

We will prove the main Theorem 1.2 in this section. Let us begin from the following Lemma.

Lemma 3.1. Let $(X, d, \mu)$ be a $R C D^{*}(K, \infty)$ space with some $K \leqslant 0$. Assume that $u(x, t): X \times$ $[0, T] \rightarrow \mathbb{R}, T \leqslant \infty$, is a solution of heat equation $\partial_{t} u=\Delta u$ with initial value $u_{0}(x) \in L^{2}(X) \cap$ $L^{\infty}(X)$ such that $u_{0} \geqslant 0$. For any $\epsilon>0$, we have $\frac{|\nabla u|^{2}}{u+\epsilon} \in \mathscr{D}\left(\Delta^{*}\right)$ for all $t \in(0, T]$ and

$$
\Delta^{*}\left(\frac{|\nabla u|^{2}}{u+\epsilon}\right)-\frac{\partial}{\partial t}\left(\frac{|\nabla u|^{2}}{u+\epsilon}\right) \cdot \mu \geqslant 2 K \frac{|\nabla u|^{2}}{u+\epsilon} \cdot \mu .
$$

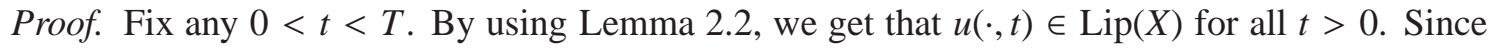
$u_{0} \in L^{2}(X) \cap L^{\infty}(X)$, we get $u \in L^{\infty}(X) \cap \mathscr{D}\left(\Delta_{\mathscr{E}}\right) \cap \operatorname{Lip}(X)$ and $\Delta u \in W^{1,2}(X)$ for all $t>0$.

Fix any $\epsilon>0$ and any $\ell>0$, we first claim that $(u+\epsilon)^{-\ell} \in \mathscr{D}(\Delta) \cap \operatorname{Lip}(X)$. Since $u \in \operatorname{Lip}(X)$ and $u+\epsilon \geqslant \epsilon$, we have $(u+\epsilon)^{-\ell} \in \operatorname{Lip}(X)$. By using Lemma2.1 (i), we have $(u+\epsilon)^{-\ell} \in \mathscr{D}\left(\Delta^{*}\right)$ and

$$
\Delta^{*}(u+\epsilon)^{-\ell}=-\ell(u+\epsilon)^{-\ell-1} \Delta^{*} u+\ell(\ell+1) \cdot(u+\epsilon)^{-\ell-2}|\nabla u|^{2} \cdot \mu .
$$

Since $\Delta^{*} u=\Delta u \cdot \mu$ with $\Delta u \in L^{2}(X),|\nabla u| \in L^{\infty}(X)$ and $(u+\epsilon)^{-\ell} \in L^{\infty}(X)$, we have $(u+\epsilon)^{-\ell} \in \mathscr{D}(\Delta)$.

By using $u \in L^{\infty}(X) \cap \mathscr{D}\left(\Delta_{\mathscr{E}}\right) \cap \operatorname{Lip}(X)$ and $\Delta u \in W^{1,2}(X)$ and Lemma 2.3, we have $|\nabla u|^{2} \in$ $\mathscr{D}\left(\Delta^{*}\right) \cap W^{1,2}(X)$. According to Lemma2.1 (ii) (with condition (c)), we conclude that $\frac{|\nabla u|^{2}}{(u+\epsilon)^{\ell}} \in \mathscr{D}\left(\Delta^{*}\right)$ for all $t>0$ and

$$
\Delta^{*}\left(\frac{|\nabla u|^{2}}{(u+\epsilon)^{\ell}}\right)=\frac{\Delta^{*}\left(|\nabla u|^{2}\right)}{(u+\epsilon)^{\ell}}+|\nabla u|^{2} \cdot \Delta^{*}\left[(u+\epsilon)^{-\ell}\right]-2 \ell \frac{\left\langle\nabla u, \nabla|\nabla u|^{2}\right\rangle}{(u+\epsilon)^{\ell+1}} \cdot \mu .
$$

By using Lemma 2.3 and (3.2), we have

$$
\Delta^{S}\left(\frac{|\nabla u|^{2}}{(u+\epsilon)^{\ell}}\right)=\frac{\Delta^{S}\left(|\nabla u|^{2}\right)}{(u+\epsilon)^{\ell}} \geqslant 0
$$

and

$$
\Delta^{R}\left(\frac{|\nabla u|^{2}}{(u+\epsilon)^{\ell}}\right)=\frac{\Delta^{R}\left(|\nabla u|^{2}\right)}{(u+\epsilon)^{\ell}}-\ell \frac{|\nabla u|^{2} \Delta u}{(u+\epsilon)^{\ell+1}}+\ell(\ell+1) \cdot \frac{|\nabla u|^{4}}{(u+\epsilon)^{\ell+2}}-2 \ell \frac{\left\langle\nabla u, \nabla|\nabla u|^{2}\right\rangle}{(u+\epsilon)^{\ell+1}} .
$$

Note that

$$
\frac{\partial}{\partial t}\left(\frac{|\nabla u|^{2}}{(u+\epsilon)^{\ell}}\right)=\frac{\partial_{t}|\nabla u|^{2}}{(u+\epsilon)^{\ell}}-\ell \frac{|\nabla u|^{2} \partial_{t} u}{(u+\epsilon)^{\ell+1}} .
$$


Using $\Delta u=\partial_{t} u, \partial_{t}|\nabla u|^{2}:=\frac{\partial}{\partial t}|\nabla u|^{2}=2\langle\nabla u, \nabla \Delta u\rangle$ and (3.4), we have, for $\mu$-a.e. $x \in X$,

$$
\Delta^{R}\left(\frac{|\nabla u|^{2}}{(u+\epsilon)^{\ell}}\right)-\frac{\partial}{\partial t}\left(\frac{|\nabla u|^{2}}{(u+\epsilon)^{\ell}}\right)=\frac{\Delta^{R}\left(|\nabla u|^{2}\right)-2\langle\nabla u, \nabla \Delta u\rangle}{(u+\epsilon)^{\ell}}+\ell(\ell+1) \frac{|\nabla u|^{4}}{(u+\epsilon)^{\ell+2}}-2 \ell \frac{\left\langle\nabla u, \nabla|\nabla u|^{2}\right\rangle}{(u+\epsilon)^{\ell+1}}
$$

Next, we take $\ell=1$ in this proof.

At the points where $|\nabla u|=0$, using (2.4) and (3.5), we have

$$
\Delta^{R}\left(\frac{|\nabla u|^{2}}{u+\epsilon}\right)-\frac{\partial}{\partial t}\left(\frac{|\nabla u|^{2}}{u+\epsilon}\right) \geqslant 0
$$

where we have used $\left|\left\langle\nabla u, \nabla|\nabla u|^{2}\right\rangle\right| \leqslant\left.|\nabla u| \cdot|\nabla| \nabla u\right|^{2} \mid=0$.

At the points where $|\nabla u| \neq 0$, by using (3.5) for $\ell=1$, we have

$$
\Delta^{R}\left(\frac{|\nabla u|^{2}}{u+\epsilon}\right)-\frac{\partial}{\partial t}\left(\frac{|\nabla u|^{2}}{u+\epsilon}\right)=\frac{2}{u+\epsilon}\left(\frac{\Delta^{R}\left(|\nabla u|^{2}\right)-2\langle\nabla u, \nabla \Delta u\rangle}{2}+\frac{|\nabla u|^{4}}{(u+\epsilon)^{2}}-\frac{\left\langle\nabla u, \nabla|\nabla u|^{2}\right\rangle}{u+\epsilon}\right) .
$$

By using (2.5) with $N=\infty$ to the function $u$ (see also Remark 2.1), we get

$$
\frac{1}{2} \Delta^{R}\left(|\nabla u|^{2}\right) \geqslant\langle\nabla \Delta u, \nabla u\rangle+K|\nabla u|^{2}+\left(\frac{\left\langle\nabla u, \nabla|\nabla u|^{2}\right\rangle}{2|\nabla u|^{2}}\right)^{2} .
$$

By combining this and the equation (3.6), we have

$$
\Delta^{R}\left(\frac{|\nabla u|^{2}}{u+\epsilon}\right)-\frac{\partial}{\partial t}\left(\frac{|\nabla u|^{2}}{u+\epsilon}\right) \geqslant \frac{2}{u+\epsilon}\left(K|\nabla u|^{2}+\mathscr{A}\right)
$$

where

$$
\mathscr{A}:=\left(\frac{\left\langle\nabla u, \nabla|\nabla u|^{2}\right\rangle}{2|\nabla u|^{2}}\right)^{2}+\frac{|\nabla u|^{4}}{(u+\epsilon)^{2}}-\frac{\left\langle\nabla u, \nabla|\nabla u|^{2}\right\rangle}{u+\epsilon}=\left(\frac{\left\langle\nabla u, \nabla|\nabla u|^{2}\right\rangle}{2|\nabla u|^{2}}-\frac{|\nabla u|^{2}}{u+\epsilon}\right)^{2} \geqslant 0 .
$$

Hence, we have, for $\mu$-a.e. $x \in X$,

$$
\Delta^{R}\left(\frac{|\nabla u|^{2}}{u+\epsilon}\right)-\frac{\partial}{\partial t}\left(\frac{|\nabla u|^{2}}{u+\epsilon}\right) \geqslant \frac{2 K|\nabla u|^{2}}{u+\epsilon} .
$$

The combination of (3.3) and (3.7) implies the desired (3.1). The proof is complete.

Remark. In the above proof, it is crucial that the singular part is nonnegative.

Lemma 3.2. Let $(X, d, \mu)$ be a $R C D^{*}(K, \infty)$ space with some $K \leqslant 0$. Assume that $u(x, t): X \times$ $[0, T] \rightarrow \mathbb{R}, T \leqslant \infty$, is a solution of heat equation $\partial_{t} u=\Delta u$ with initial value $u_{0}(x) \in L^{2}(X) \cap$ $L^{\infty}(X)$ such that $u_{0} \geqslant 0$. For any $\epsilon>0$, we set

$$
P_{\epsilon}(x, t):=\varphi(t) \frac{|\nabla u|^{2}}{u+\epsilon}+(u+\epsilon) \log (u+\epsilon),
$$

where $\varphi(t)=\frac{t}{1-2 K t}$. Then we have $P_{\epsilon}(\cdot, t) \in \mathscr{D}\left(\Delta^{*}\right)$ for all $t \in(0, T]$ and

$$
\frac{\partial}{\partial t} P_{\epsilon}(x, t) \cdot \mu \leqslant \Delta^{*} P_{\epsilon}(x, t)
$$


Proof. From Lemma 3.1 we have $\frac{|\nabla u|^{2}}{u+\epsilon} \in \mathscr{D}\left(\Delta^{*}\right)$ for all $t>0$ and

$$
\Delta^{*}\left(\frac{|\nabla u|^{2}}{u+\epsilon}\right)-\frac{\partial}{\partial t}\left(\frac{|\nabla u|^{2}}{u+\epsilon}\right) \cdot \mu \geqslant 2 K \frac{|\nabla u|^{2}}{u+\epsilon} \cdot \mu .
$$

Since $u \in \operatorname{Lip}(X)$ and $u+\epsilon \geqslant \epsilon$, we have $(u+\epsilon) \log (u+\epsilon) \in \operatorname{Lip}(X)$. By using Lemma2.1 (i), we have $(u+\epsilon) \log (u+\epsilon) \in \mathscr{D}\left(\Delta^{*}\right)$ and

$$
\begin{aligned}
\Delta^{*}((u+\epsilon) \log (u+\epsilon)) & =(1+\log (u+\epsilon)) \Delta^{*} u+\frac{|\nabla u|^{2}}{u+\epsilon} \cdot \mu \\
& =\left[(1+\log (u+\epsilon)) \Delta u+\frac{|\nabla u|^{2}}{u+\epsilon}\right] \cdot \mu,
\end{aligned}
$$

where we have used $\Delta^{*} u=\Delta u \cdot \mu$ with $\Delta u \in L^{2}(X)$. Hence we have

$$
\Delta^{*}((u+\epsilon) \log (u+\epsilon))-\frac{\partial}{\partial t}(u+\epsilon) \log (u+\epsilon) \cdot \mu=\frac{|\nabla u|^{2}}{u+\epsilon} \cdot \mu,
$$

since $\partial_{t} u=\Delta u$. By applying (3.9) and (3.10), we have

$$
\Delta^{*} P_{\epsilon}(x, t)-\frac{\partial}{\partial t} P_{\epsilon}(x, t) \cdot \mu \geqslant\left(1-\varphi^{\prime}+2 K \varphi\right) \cdot \frac{|\nabla u|^{2}}{u+\epsilon} \cdot \mu .
$$

Together with $1-\varphi^{\prime}(t)+2 K \varphi(t)=-2 K t /(1-2 K t)^{2} \geqslant 0$ (since $K \leqslant 0$ ), we get $\Delta^{*} P_{\epsilon}(x, t)-$ $\frac{\partial}{\partial t} P_{\epsilon}(x, t) \cdot \mu \geqslant 0$. The proof is finished.

We also need the the following lemma, which can be found [15] or [31, Lemma 2 on Page 165].

Lemma 3.3. Let a function $v(x, t)$ on $X \times[0, \infty)$ be a weak sub-solution of the heat equation and $v(x, 0)=v_{0}(x)$, i.e., $v(x, t)$ satisfies $\partial_{t} v \leqslant \Delta v$ in the sense of distribution. Namely, the following inequality

$$
-\int_{0}^{\infty} \int_{X}\langle\nabla v, \nabla \varphi\rangle d \mu d t \geqslant \int_{0}^{\infty} \int_{X} \partial_{t} v \cdot \varphi d \mu d t
$$

holds for any nonnegative function $\varphi(x, t) \in \operatorname{Lip}_{0}(X \times[0, \infty))$.

Let a function $g(x, t)$ be of class $C^{1}$ in $t$ and Lipschitz continuous in $x$, and satisfy

$$
g \leqslant 0, \quad \frac{\partial}{\partial t} g+\frac{1}{4}|\nabla g|^{2}=0 .
$$

For any $T>0$, if we assume

$$
\int_{0}^{T} \int_{X} e^{\frac{g(x, t)}{4}} \cdot v^{2}(x, t) d \mu d t<\infty
$$

then, for any $R>0$ and $x_{0} \in X$, we have

$$
\int_{B\left(x_{0}, R\right)} e^{\frac{g(x, T)}{4}} v^{2}(x, T) d \mu \leqslant \int_{X} e^{\frac{g(x, 0)}{4}} v_{0}^{2}(x) d \mu .
$$

Proof. See the proof in [31, Page 166-167] and take $\epsilon=4$ there. 
Now we are in the position to prove the main theorem.

Proof of Theorem 1.2 By replacing $u$ by $u / 2 M$, we can assume that $0<u \leqslant 1 / 2$.

(i). First, we consider the case where the initial data $u_{0} \in L^{2}(X)$. For an arbitrarily fixed $\epsilon \epsilon$ $(0,1 / 2)$, set $u_{\epsilon}:=u+\epsilon$ and

$$
P_{\epsilon}(x, t):=\varphi(t) \frac{|\nabla u|^{2}}{u_{\epsilon}}+u_{\epsilon} \log \left(u_{\epsilon}\right)
$$

where $\varphi(t):=t /(1-2 K t)$. By Lemma3.2, we have

$$
\frac{\partial}{\partial t} P_{\epsilon} \cdot \mu \leqslant \Delta^{*} P_{\epsilon}
$$

and hence $P_{\epsilon}^{+}$is a weak sub-solution of the heat equation, where $P_{\epsilon}^{+}(x, t):=\max \left\{P_{\epsilon}(x, t), 0\right\}$.

Fix a $T>0$. The volume growth condition (2.2) implies that there exists a small constant $\delta=\delta\left(c_{1}, c_{2}\right)>0$ such that

$$
\int_{X} e^{-\frac{d^{2}\left(x_{0}, x\right)}{\delta}} d \mu<\infty
$$

for some $x_{0} \in X$. We denote

$$
g(x, t):=-\frac{d^{2}\left(x_{0}, x\right)}{T-t+\delta / 4} \leqslant-\frac{d^{2}\left(x_{0}, x\right)}{\delta / 4} .
$$

Then

From Lemma2.2, we have

$$
\frac{\partial}{\partial t} g+\frac{1}{4}|\nabla g|^{2}=0
$$

$$
P_{\epsilon}^{+} \leqslant \varphi(t) \frac{|\nabla u|^{2}}{u_{\epsilon}} \leqslant \varphi(t) \cdot \frac{C^{\prime}}{t} \cdot \epsilon^{-1} \leqslant C^{\prime} \epsilon^{-1}
$$

for some constant $C^{\prime}=C^{\prime}(K, T)>0$, where we have used $K \leqslant 0$ and $u, u_{\epsilon} \leqslant 1$. Hence,

$$
\int_{0}^{T} \int_{X} e^{\frac{g(x, t)}{4}}\left(P_{\epsilon}^{+}\right)^{2}(x, t) d \mu \leqslant T \cdot\left(C^{\prime} \epsilon^{-1}\right)^{2} \cdot \int_{X} e^{-\frac{d^{2}\left(x_{0}, x\right)}{\delta}} d \mu<\infty .
$$

Now the functions $P_{\epsilon}^{+}$and $g$ meet all of assumptions of Lemma 3.3 . Hence we conclude that

$$
\int_{B\left(x_{0}, R\right)}\left(P_{\epsilon}^{+}(x, T)\right)^{2} \cdot e^{g / 4} d \mu \leqslant \int_{X}\left(P_{\epsilon}^{+}(x, 0)\right)^{2} \cdot e^{g / 4} d \mu .
$$

Notice that $P_{\epsilon}^{+}(x, 0)=0$, we have obtained, from equation (3.12),

$$
\int_{B\left(x_{0}, R\right)}\left(P_{\epsilon}^{+}(x, T)\right)^{2} \cdot e^{g / 4} d \mu=0, \quad \text { and then, } \quad P_{\epsilon}^{+}(x, T)=0 .
$$

Therefore, we have $P_{\epsilon}(x, T) \leqslant 0$. That is,

$$
\varphi(T) \frac{|\nabla u|^{2}}{u_{\epsilon}^{2}} \leqslant-\log u_{\epsilon} .
$$


Letting $\epsilon \rightarrow 0^{+}$, we have

$$
\varphi(T) \frac{|\nabla u|^{2}}{u^{2}} \leqslant \log \left(\frac{1}{u}\right)
$$

This proves the desired Hamilton estimate for the case where the initial data $u_{0} \in L^{2}(X)$.

(ii). The proof of the case where the initial data $u_{0} \in L^{q}(X)$ for some $q \in[1, \infty)$.

It is sufficient to consider the case where $q \geqslant 2$. Indeed, if $q<2$, the facts $u_{0}(x) \in L^{q}(X)$ and $0 \leqslant u(x, t) \leqslant M$ implies that $u_{0} \in L^{2}(X)$. Take a sequence of functions $\tilde{u}_{j} \in L^{2}(X) \cap L^{\infty}(X)$ such that

$$
\tilde{u}_{j} \stackrel{L^{q}}{\rightarrow} u_{0}, \quad \tilde{u}_{j} \stackrel{w-L^{\infty}}{\rightarrow} u_{0} \quad \text { and } \quad 0 \leqslant \tilde{u}_{j} \leqslant M .
$$

Fix an $\epsilon>0$ and let $u_{j}(x, t):=H_{t} \tilde{u}_{j}(x)$ and $u_{j, \epsilon}:=u_{j}+\epsilon$. Now we have

$$
\epsilon \leqslant u_{j, \epsilon}(x, t) \leqslant M+\epsilon, j=1,2, \cdots .
$$

For any fixed $T>0, R>0$, and any Lipschitz function $0 \leqslant f(x) \in \operatorname{Lip}_{0}\left(B_{R}\left(x_{0}\right)\right)$, from (3.13), we get

$$
\int_{B_{R}\left(x_{0}\right)} \varphi(T)\left|\nabla u_{j}\right|^{2} \cdot f d \mu \leqslant-\int_{B_{R}\left(x_{0}\right)} u_{j, \epsilon}^{2} \cdot \log u_{j, \epsilon} \cdot f d \mu, \quad j=1,2, \cdots
$$

The fact $\tilde{u}_{j} \stackrel{L^{q}}{\rightarrow} u_{0}$ implies that $u_{j}(x, T) \stackrel{L^{q}}{\rightarrow} u(x, T)$ by $L^{q}$-boundedness of $H_{t}$. Thus, $u_{j}(x, T) \rightarrow$ $u(x, T)$ for almost all $x \in X$, up to a subsequence. Notice that $u_{j, \epsilon}^{2} \cdot \log u_{j, \epsilon}$ is uniformly bounded on $B_{R}\left(x_{0}\right)$, by dominated convergence theorem, we have

$$
\lim _{j \rightarrow \infty} \int_{B_{R}\left(x_{0}\right)} u_{j, \epsilon}^{2} \cdot \log u_{j, \epsilon} \cdot f d \mu=\int_{B_{R}\left(x_{0}\right)} u_{\epsilon}^{2} \cdot \log u_{\epsilon} \cdot f d \mu .
$$

Notice that $q \geqslant 2$ and $u_{j}(x, T) \stackrel{L^{q}}{\rightarrow} u(x, T)$, it is clear that $u_{j}(x, T) \stackrel{L^{2}\left(B_{R}\left(x_{0}\right)\right)}{\rightarrow} u(x, T)$. Therefore, by the lower semi-continuity of energy and (3.14)-3.15), we have

$$
\int_{B_{R}\left(x_{0}\right)} \varphi(T)|\nabla u|^{2} \cdot f d \mu \leqslant \liminf _{j \rightarrow \infty} \int_{B_{R}\left(x_{0}\right)} \varphi(T)\left|\nabla u_{j}\right|^{2} \cdot f d \mu \leqslant-\int_{B_{R}\left(x_{0}\right)} u_{\epsilon}^{2} \cdot \log u_{\epsilon} \cdot f d \mu .
$$

By the arbitrariness of $f$ and $R$, we get $\varphi(T)|\nabla u|^{2} \leqslant-u_{\epsilon}^{2} \cdot \log u_{\epsilon}$. Letting $\epsilon \rightarrow 0^{+}$, we have

$$
\varphi(T)|\nabla u|^{2} \leqslant u^{2} \cdot \log \left(\frac{1}{u}\right) .
$$

This is the desired Hamilton estimate.

\section{Monotonicity for heat equations}

We will prove the main Theorem 1.3 in this section. 
Lemma 4.1. Let $(X, d, \mu)$ be a proper $R C D^{*}(0, N)$ space with some $N \in[1, \infty]$. Assume that $u(x, t): X \times[0, T] \rightarrow \mathbb{R}, T \leqslant \infty$, is a solution of heat equation $\partial_{t} u=\Delta u$ with initial value $0 \leq u_{0}(x) \in L^{2}(X) \cap L^{\infty}(X)$. For any $\epsilon>0$, we have $w_{\epsilon}(x, t) \in \mathscr{D}\left(\Delta^{*}\right)$ for all $t \in(0, T]$ and

$$
\Delta^{*} w_{\epsilon}-\frac{\partial}{\partial t} w_{\epsilon} \cdot \mu \geqslant\left[-2 \frac{\left\langle\nabla w_{\epsilon}, \nabla u\right\rangle}{u_{\epsilon}}+\frac{2}{N u_{\epsilon}^{2}}\left(\Delta u-\frac{|\nabla u|^{2}}{u_{\epsilon}}\right)^{2}\right] \cdot \mu
$$

where $u_{\epsilon}:=u+\epsilon$ and

$$
w_{\epsilon}(x, t):=\frac{|\nabla u|^{2}}{u_{\epsilon}^{2}}-2 \frac{\partial_{t} u}{u_{\epsilon}} .
$$

Here and in the sequel, if $N=\infty$, then $\frac{1}{N}=0$ and $\frac{N-1}{N}=1$.

Proof. By (3.3) and (3.5) the proof in the Lemma 3.1 and taking $\ell=2$ there, we have $|\nabla u|^{2} / u_{\epsilon}^{2} \in$ $\mathscr{D}\left(\Delta^{*}\right)$ with

$$
\Delta^{S}\left(|\nabla u|^{2} / u_{\epsilon}^{2}\right) \geqslant 0
$$

and, for $\mu$-a.e. $x \in X$,

$$
\Delta^{R}\left(\frac{|\nabla u|^{2}}{u_{\epsilon}^{2}}\right)-\frac{\partial}{\partial t}\left(\frac{|\nabla u|^{2}}{u_{\epsilon}^{2}}\right)=\frac{\Delta^{R}\left(|\nabla u|^{2}\right)-2\langle\nabla u, \nabla \Delta u\rangle}{u_{\epsilon}^{2}}+6 \frac{|\nabla u|^{4}}{u_{\epsilon}^{4}}-4 \frac{\left\langle\nabla u, \nabla|\nabla u|^{2}\right\rangle}{u_{\epsilon}^{3}} .
$$

Since $\partial_{t} u$ also solves the heat equation, we have $\partial_{t} u \in \operatorname{Lip}(X)$. From Lemma 2.1(ii) (with condition (a)), we have $\frac{\partial_{t} u}{u_{\epsilon}} \in \mathscr{D}\left(\Delta^{*}\right)$ and

$$
\Delta^{*}\left(\frac{\partial_{t} u}{u_{\epsilon}}\right)=\Delta^{*}\left(\partial_{t} u\right) \cdot u_{\epsilon}^{-1}+\partial_{t} u\left(-u_{\epsilon}^{-2} \cdot \Delta^{*} u+2 u_{\epsilon}^{-3}|\nabla u|^{2} \cdot \mu\right)-2\left\langle\nabla \partial_{t} u, u_{\epsilon}^{-2} \nabla u\right\rangle \cdot \mu .
$$

By noting $\Delta^{*} u=\partial_{t} u \cdot \mu$ and $\Delta^{*}\left(\partial_{t} u\right)=\Delta\left(\partial_{t} u\right) \cdot \mu$, we have

$$
\Delta^{*}\left(\frac{\partial_{t} u}{u_{\epsilon}}\right)-\frac{\partial}{\partial t}\left(\frac{\partial_{t} u}{u_{\epsilon}}\right) \cdot \mu=2 \partial_{t} u \cdot u_{\epsilon}^{-3}|\nabla u|^{2} \cdot \mu-2 u_{\epsilon}^{-2}\left\langle\nabla \partial_{t} u, \nabla u\right\rangle \cdot \mu .
$$

Now $w_{\epsilon} \in \mathscr{D}\left(\Delta^{*}\right) \subset W_{\text {loc }}^{1,2}(X)$, we have

$$
\left\langle\nabla w_{\epsilon}, \nabla u\right\rangle=u_{\epsilon}^{-2}\left\langle\nabla|\nabla u|^{2}, \nabla u\right\rangle-2 u_{\epsilon}^{-3}|\nabla u|^{4}-2 u_{\epsilon}^{-1}\left\langle\nabla \partial_{t} u, \nabla u\right\rangle+2 u_{\epsilon}^{-2} \partial_{t} u \cdot|\nabla u|^{2} .
$$

Therefore, by combining with (4.2), (4.3) and (4.4), we obtain that $\Delta^{S} w_{\epsilon} \geqslant 0$ and, for $\mu$-a.e. $x \in X$,

$$
\begin{aligned}
\Delta^{R} w_{\epsilon}- & \frac{\partial}{\partial t} w_{\epsilon}+2 \frac{\left\langle\nabla w_{\epsilon}, \nabla u\right\rangle}{u_{\epsilon}} \\
= & \frac{\Delta^{R}\left(|\nabla u|^{2}\right)-2\langle\nabla u, \nabla \Delta u\rangle}{u_{\epsilon}^{2}}+6 \frac{|\nabla u|^{4}}{u_{\epsilon}^{4}}-4 \frac{\left\langle\nabla u, \nabla|\nabla u|^{2}\right\rangle}{u_{\epsilon}^{3}} \\
& -2\left[2 \partial_{t} u \cdot u_{\epsilon}^{-3}|\nabla u|^{2}-2 u_{\epsilon}^{-2}\left\langle\nabla \partial_{t} u, \nabla u\right\rangle\right] \\
& +2\left[u_{\epsilon}^{-3}\left\langle\nabla|\nabla u|^{2}, \nabla u\right\rangle-2 u_{\epsilon}^{-4}|\nabla u|^{4}-2 u_{\epsilon}^{-2}\left\langle\nabla \partial_{t} u, \nabla u\right\rangle+2 u_{\epsilon}^{-3} \partial_{t} u \cdot|\nabla u|^{2}\right] \\
= & \frac{\Delta^{R}\left(|\nabla u|^{2}\right)-2\langle\nabla u, \nabla \Delta u\rangle}{u_{\epsilon}^{2}}+2 \frac{|\nabla u|^{4}}{u_{\epsilon}^{4}}-2 \frac{\left\langle\nabla u, \nabla|\nabla u|^{2}\right\rangle}{u_{\epsilon}^{3}} .
\end{aligned}
$$


At the points where $|\nabla u|=0$, using (2.4) and (4.5), we have

$$
\Delta^{R} w_{\epsilon}-\frac{\partial}{\partial t} w_{\epsilon}+2 \frac{\left\langle\nabla w_{\epsilon}, \nabla u\right\rangle}{u_{\epsilon}} \geqslant \frac{2}{N u_{\epsilon}^{2}}(\Delta u)^{2},
$$

where we have used $\left|\left\langle\nabla u, \nabla|\nabla u|^{2}\right\rangle\right| \leqslant\left.|\nabla u| \cdot|\nabla| \nabla u\right|^{2} \mid=0$.

At the points where $|\nabla u| \neq 0$, using (2.5) and (4.5), we have

$$
\Delta^{R} w_{\epsilon}-\frac{\partial}{\partial t} w_{\epsilon}+2 \frac{\left\langle\nabla w_{\epsilon}, \nabla u\right\rangle}{u_{\epsilon}} \geqslant \frac{2}{u_{\epsilon}^{2}} \cdot \mathscr{A},
$$

where

$$
\mathscr{A}:=\frac{(\Delta u)^{2}}{N}+\frac{N}{N-1}\left(\frac{\left\langle\nabla u, \nabla|\nabla u|^{2}\right\rangle}{2|\nabla u|^{2}}-\frac{\Delta u}{N}\right)^{2}+\frac{|\nabla u|^{4}}{u_{\epsilon}^{2}}-\frac{\left\langle\nabla u, \nabla|\nabla u|^{2}\right\rangle}{u_{\epsilon}} .
$$

Let us set $B_{1}:=\frac{\left\langle\nabla u, \nabla|\nabla u|^{2}\right\rangle}{|\nabla u|^{2}}$ and $B_{2}:=\frac{|\nabla u|^{2}}{u_{\epsilon}}$. We get

$$
\begin{aligned}
\mathscr{A} & =\frac{(\Delta u)^{2}}{N}+\frac{N}{N-1}\left(\frac{B_{1}^{2}}{4}-\frac{B_{1} \cdot \Delta u}{N}+\frac{(\Delta u)^{2}}{N^{2}}\right)+B_{2}^{2}-B_{1} B_{2} \\
& =\frac{(\Delta u)^{2}}{N-1}+\frac{N}{N-1}\left[\frac{B_{1}^{2}}{4}-B_{1} \cdot\left(\frac{\Delta u}{N}+\frac{N-1}{N} B_{2}\right)\right]+B_{2}^{2} \\
& \geqslant \frac{(\Delta u)^{2}}{N-1}+B_{2}^{2}+\frac{N}{N-1}\left[-\left(\frac{\Delta u}{N}+\frac{N-1}{N} B_{2}\right)^{2}\right] \\
& =\frac{(\Delta u)^{2}}{N-1}+B_{2}^{2}-\left(\frac{(\Delta u)^{2}}{N(N-1)}+\frac{2 B_{2} \cdot \Delta u}{N}+\frac{N-1}{N} B_{2}^{2}\right) \\
& =\frac{\left(\Delta u-B_{2}\right)^{2}}{N} .
\end{aligned}
$$

Therefore, we have

$$
\Delta^{R} w_{\epsilon}-\frac{\partial}{\partial t} w_{\epsilon}+2 \frac{\left\langle\nabla w_{\epsilon}, \nabla u\right\rangle}{u_{\epsilon}} \geqslant \frac{2}{N u_{\epsilon}^{2}} \cdot\left(\Delta u-B_{2}\right)^{2} .
$$

Together with $\Delta^{S} w_{\epsilon} \geqslant 0$, we have proved the lemma.

Lemma 4.2. Let $(X, d, \mu)$ be a $R C D^{*}(0, N)$ space with some $N \in[1, \infty)$. Assume that $u(x, t)$ : $X \times[0, T] \rightarrow \mathbb{R}, T \leqslant \infty$, is a solution of heat equation $\partial_{t} u=\Delta u$ with initial value $u_{0}(x) \in$ $L^{2}(X) \cap L^{\infty}(X)$ such that $u_{0} \geqslant 0$. For each $\epsilon>0$, we set

$$
W_{\epsilon}(x, t):=\tau \cdot w_{\epsilon}-\log u_{\epsilon}-\frac{N}{2} \log (4 \pi \tau)-N,
$$

where $\tau=\tau(t)>0$ with $\frac{d \tau}{d t}=1$ and $u_{\epsilon}, w_{\epsilon}$ are given in Lemma 4.1 Then $W_{\epsilon} \in \mathscr{D}\left(\Delta^{*}\right)$ and

$$
\Delta^{*} W_{\epsilon}-\frac{\partial}{\partial t} W_{\epsilon} \cdot \mu \geqslant-2 \frac{\left\langle\nabla W_{\epsilon}, \nabla u\right\rangle}{u_{\epsilon}} \cdot \mu .
$$


Proof. From Lemma 4.1 and that $\log u_{\epsilon} \in \mathscr{D}\left(\Delta^{*}\right)$, we deduce that $W_{\epsilon} \in \mathscr{D}\left(\Delta^{*}\right)$. Moreover, $\Delta^{S} W_{\epsilon}=$ $\tau \cdot \Delta^{S} w_{\epsilon} \geqslant 0$. By directly calculating and using Lemma4.1, we get

$$
\begin{aligned}
\left(\Delta^{R}-\frac{\partial}{\partial t}\right) W_{\epsilon} & =\tau \cdot\left(\Delta^{R}-\frac{\partial}{\partial t}\right) w_{\epsilon}-w_{\epsilon}+\frac{|\nabla u|^{2}}{u_{\epsilon}^{2}}+\frac{N}{2 \tau} \\
& \geqslant-2 \frac{\left\langle\nabla\left(\tau \cdot w_{\epsilon}\right), \nabla u\right\rangle}{u_{\epsilon}}+\frac{2 \cdot \tau}{N u_{\epsilon}^{2}}\left(\Delta u-\frac{|\nabla u|^{2}}{u_{\epsilon}}\right)^{2}-\frac{|\nabla u|^{2}}{u_{\epsilon}^{2}}+2 \frac{\Delta u}{u_{\epsilon}}+\frac{|\nabla u|^{2}}{u_{\epsilon}^{2}}+\frac{N}{2 \tau} \\
& =-2 \frac{\left\langle\nabla\left(W_{\epsilon}+\log u_{\epsilon}\right), \nabla u\right\rangle}{u_{\epsilon}}+\frac{2 \cdot \tau}{N}\left(\frac{\Delta u}{u_{\epsilon}}-\frac{|\nabla u|^{2}}{u_{\epsilon}^{2}}\right)^{2}+2 \frac{\Delta u}{u_{\epsilon}}+\frac{N}{2 \tau} \\
& =-2 \frac{\left\langle\nabla W_{\epsilon}, \nabla u\right\rangle}{u_{\epsilon}}+\frac{2 \cdot \tau}{N}\left(\frac{\Delta u}{u_{\epsilon}}-\frac{|\nabla u|^{2}}{u_{\epsilon}^{2}}\right)^{2}+2\left(\frac{\Delta u}{u_{\epsilon}}-\frac{|\nabla u|^{2}}{u_{\epsilon}^{2}}\right)+\frac{N}{2 \tau} \\
& \geqslant-2 \frac{\left\langle\nabla W_{\epsilon}, \nabla u\right\rangle}{u_{\epsilon}} .
\end{aligned}
$$

Together with $\Delta^{S} W_{\epsilon} \geqslant 0$, we have proved the lemma.

Now let us prove Theorem 1.3 .

Proof of Theorem 1.3

(i) The case $N=\infty$. The assumption that $X$ is compact and $u_{0} \in L^{\infty}(X)$ implies that $\mu(X)<\infty$ and $u_{0} \in L^{2}(X)$. Fix an $\epsilon>0$ and set $u_{\epsilon}=u+\epsilon$. By using Lemma 4.1, we have

$$
\begin{aligned}
\Delta^{*}\left(w_{\epsilon} u_{\epsilon}\right)-\frac{\partial}{\partial t}\left(w_{\epsilon} u_{\epsilon}\right) \cdot \mu & =u_{\epsilon}\left(\Delta^{*} w_{\epsilon}-\frac{\partial}{\partial t} w_{\epsilon} \cdot \mu\right)+2\left\langle\nabla w_{\epsilon}, \nabla u\right\rangle \cdot \mu \\
& \geqslant-2\left\langle\nabla w_{\epsilon}, \nabla u\right\rangle \cdot \mu+2\left\langle\nabla w_{\epsilon}, \nabla u\right\rangle \cdot \mu=0 .
\end{aligned}
$$

Moreover, notice that since $\mu(X)<\infty$, we have that $|\nabla u|^{2} \in W^{1,2} \cap L^{\infty}(X)$ and $u_{\epsilon}^{-1} \in \operatorname{Lip}(X)$, and $1 \in W^{1,2}(X)$ is an admissible test function for the measure $\Delta^{*}\left(|\nabla u|^{2} / u_{\epsilon}\right)$ and $\Delta u$. From these, we conclude that $\int_{X} \Delta u=0$ and $\int_{X} \Delta^{*}\left(w_{\epsilon} u_{\epsilon}\right) d \mu=0$, and therefore

$$
\int_{X} w_{\epsilon} u_{\epsilon} d \mu=\int_{X}\left(|\nabla u|^{2} / u_{\epsilon}-2 \Delta u\right) d \mu=\int_{X}|\nabla u|^{2} / u_{\epsilon} d \mu
$$

is monotone decreasing, since $\int_{X} \frac{\partial}{\partial t}\left(w_{\epsilon} u_{\epsilon}\right) d \mu \leq \int_{X} \Delta^{*}\left(w_{\epsilon} u_{\epsilon}\right) d \mu=0$.

Note that $u(\cdot, t)$ is positive and Lipschitz continuous on $X$ for any $t>0$ and $X$ is compact. Then $f=-\log u$ is also Lipschitz continuous on $X$ for any $t>0$. Therefore,

$$
\lim _{\epsilon \rightarrow 0^{+}} \int_{X} w_{\epsilon} u_{\epsilon} d \mu=\lim _{\epsilon \rightarrow 0^{+}} \int_{X}|\nabla f|^{2} \cdot \frac{u^{2}}{u_{\epsilon}} d \mu=\int_{X}|\nabla f|^{2} u d \mu=\mathcal{W}_{\infty}(f, t)
$$

for any $t>0$. Let $\epsilon \rightarrow 0^{+}$, we conclude that $\mathcal{W}_{\infty}(f, t)$ is monotone decreasing.

(ii) The case of $N<\infty$. Since $\mu(X)<\infty$, we have $u_{0} \in L^{2}(X)$. Fix any $\epsilon>0$. Using Lemma 4.2, we have

$$
\begin{aligned}
\Delta^{*}\left(W_{\epsilon} u_{\epsilon}\right)-\frac{\partial}{\partial t}\left(W_{\epsilon} u_{\epsilon}\right) \cdot \mu & =u_{\epsilon}\left(\Delta^{*} W_{\epsilon}-\frac{\partial}{\partial t} W_{\epsilon} \cdot \mu\right)+2\left\langle\nabla W_{\epsilon}, \nabla u\right\rangle \cdot \mu \\
& \geqslant-2\left\langle\nabla W_{\epsilon}, \nabla u\right\rangle \cdot \mu+2\left\langle\nabla W_{\epsilon}, \nabla u\right\rangle \cdot \mu=0 .
\end{aligned}
$$


This and the same argument as in (i) imply that $\int_{X} W_{\epsilon} u_{\epsilon} d \mu$ is monotone decreasing. By the definition of $W_{\epsilon}$, we have

$$
\begin{aligned}
\int_{X} W_{\epsilon} u_{\epsilon} d \mu & =\int_{X} \tau\left(\frac{|\nabla u|^{2}}{u_{\epsilon}}-\partial_{t} u\right)-\left[\log \left(u_{\epsilon} \cdot(2 \pi \tau)^{N / 2}\right)+N\right] u_{\epsilon} d \mu \\
& =\int_{X} \tau \frac{|\nabla u|^{2}}{u_{\epsilon}}-\left[\log \left(u_{\epsilon} \cdot(2 \pi \tau)^{N / 2}\right)+N\right] u_{\epsilon} d \mu
\end{aligned}
$$

The same argument in (i) implies that $f=-\log \left(u(4 \pi \tau)^{N / 2}\right)$ is Lipschitz continuous on $X$ for any $t>0$. Therefore,

$$
\lim _{\epsilon \rightarrow 0^{+}} \int_{X} W_{\epsilon} u_{\epsilon} d \mu=\int_{X}\left(\tau|\nabla f|^{2}+f-N\right) u d \mu=W_{N}(f, \tau)
$$

for any $t>0$. Let $\epsilon \rightarrow 0^{+}$, we conclude that $\mathcal{W}_{N}(f, t)$ is monotone decreasing. Now we complete the proof.

\section{References}

[1] L. Ambrosio, N. Gigli, A. Mondino, T. Rajala, Riemannian Ricci curvature lower bounds in metric measure spaces with $\sigma$-finite measure, Trans. Amer. Math. Soc. 367 (2015), no.7, 4661-4701.

[2] L. Ambrosio, N. Gigli, G. Savaré, Metric measure spaces with Riemannian Ricci curvature bounded from below, Duke Math. J. 163 (2014), 1405-1490.

[3] L. Ambrosio, N. Gigli, G. Savaré, Bakry-Émery curvature-dimension condition and Riemannian Ricci curvature bounds, Ann. Probab. 43 (2015), 339-404.

[4] L. Ambrosio, N. Gigli, G. Savaré, Density of Lipschitz functions and equivalence of weak gradients in metric measure spaces, Rev. Mat. Iberoam. 29 (2013), 969-996.

[5] L. Ambrosio, A. Mondino, G. Savaré, On the Bakry-Émery condition, the gradient estimates and the Local-to-Global property of $\operatorname{RCD}^{*}(K, N)$ metric measure spaces, J. Geom. Anal.( to appear), DOI: 10.1007/s12220-014-9537-7.

[6] L. Ambrosio, A. Mondino, G. Savaré, Nonlinear diffusion equations and curvature conditions in metric measure spaces, 2013, Preprint.

[7] D. Bakry, F. Bolley \& I. Gentil, The Li-Yau inequality and applications under a curvaturedimension condition, avilable at http://arxiv.org/abs/1412.5165.

[8] D. Bakry, Z. Qian, Some new results on eigenvectors via dimension, diameter, and Ricci curvature, Adv. Math. 155 (2000), 98-153.

[9] J. Cheeger, Differentiability of Lipschitz functions on metric measure spaces, Geom. Funct. Anal. 9 (1999), 428-517.

[10] T.H. Colding, New monotonicity formulas for Ricci curvature and applications. I, Acta Math. 209 (2012), no. 2, 229-263.

[11] M. Erbar, K. Kuwada, K.T. Sturm, On the equivalence of the entropic curvature-dimension condition and Bochner's inequality on metric measure spaces, Invent. math. (to appear), DOI 10.1007/s00222-014-0563-7. 
[12] N. Garofalo, A. Mondino, Li-Yau and Harnack type inequalities in $R C D^{*}(K, N)$ metric measure spaces, Nonlinear Anal. 95 (2014), 721-734.

[13] N. Gigli, On the differential structure of metric measure spaces and applications, Mem. Amer. Math. Soc., Volume 236, Number 1113.

[14] N. Gigli, A. Mondino, A PDE approach to non linear potential theory, J. Math. Pures Appl. 100(4) (2013), 505-534.

[15] A. Grigor'yan, On stochastically complete manifolds, Soviet Math. Dokl., 34 (1987), 310313.

[16] R. Hamilton, A matrix Harnack estimate for the heat equation, Comm. Anal. Geom. 1 (1993), 113-126.

[17] B. B. Hua, M. Kell, C. Xia, Harmonic functions on metric measure spaces, arXiv:1308.3607.

[18] R. Jiang, Cheeger-harmonic functions in metric measure spaces revisited, J. Funct. Anal. 266 (2014), 1373-1394.

[19] R. Jiang, The Li-Yau inequality and heat kernels on metric measure spaces, J. Math. Pures Appl. 104(1) (2015), 29-57.

[20] B.L. Kotschwar, Hamilton's gradient estimate for the heat kernel on complete manifolds, Proc. Amer. Math. Soc. 135 (2007), 3013-3019.

[21] J.F. Li, X.J. Xu, Differential Harnack inequalities on Riemannian manifolds I: linear heat equation, Adv. Math. 226 (2011), 4456-4491.

[22] P. Li, Uniqueness of $L^{1}$ solutions for the Laplace equation and the heat equation on Riemannian manifolds. J. Differential Geom. 20 (1984), 447-457.

[23] P. Li, S.T. Yau, On the parabolic kernel of the Schrödinger operator, Acta Math. 156 (1986), 153-201.

[24] J. Lott, C. Villani, Ricci curvature for metric-measure spaces via optimal transport, Ann. of Math. (2) 169 (2009), 903-991.

[25] L. Ni, The entropy formula for linear heat equation J. Geom. Anal. 14 (2004), 87-100.

[26] A. Petrunin, Alexandrov meets Lott-Villani-Sturm, Münster J. of Math. 4 (2011), 53-64.

[27] P. Souplet, Q.S. Zhang, Sharp gradient estimate and Yau's Liouville theorem for the heat equation on noncompact manifolds, Bull. London Math. Soc. 38 (2006), 1045-1053.

[28] B. Qian, A generalization of Hamilton's gradient estimate, J. Geom. Phys. 62 (2012), 1064 1069.

[29] Z.H. Qian, H.C. Zhang, X.-P. Zhu, Sharp spectral gap and Li-Yau's estimate on Alexandrov spaces, Math. Z. 273 (2013), 1175-1195.

[30] G. Savaré, Self-improvement of the Bakry-Émery condition and Wasserstein contraction of the heat flow in $R C D(K, \infty)$ metric measure spaces, Discrete Contin. Dyn. Syst. 34 (2014), 1641-1661.

[31] R. Schoen, S.T. Yau, Lectures on differential geometry, International Press, Boston, 1994.

[32] N. Shanmugalingam, Newtonian spaces: an extension of Sobolev spaces to metric measure spaces, Rev. Mat. Iberoamericana 16 (2000), 243-279.

[33] S. Strichartz, Analysis of the Laplacian on the complete Riemannian manifold. J. Funct. Anal. 52 (1983), 48-79.

[34] K.T. Sturm, On the geometry of metric measure spaces I, Acta Math. 196 (2006), 65-131.

[35] K.T. Sturm, On the geometry of metric measure spaces II, Acta Math. 196 (2006), 133-177. 
[36] L. F. Wang, Monotonicity formulas via the Bakry-Émery curvature, Nonlinear Anal., 89 (2013), 230-241.

[37] J.Y. Wu, Sharp Hamilton's Laplacian estimate for the heat kernel on complete manifolds, Proc. Amer. Math. Soc. 141 (2013), 4401-4409.

[38] H.C. Zhang, X.-P. Zhu, Yau's gradient estimates on Alexandrov spaces, J. Differential Geom. 91 (2012), 445-522.

\section{Renjin Jiang}

School of Mathematical Sciences, Beijing Normal University, Laboratory of Mathematics and Complex Systems, Ministry of Education, 100875, Beijing, China

E-mail address: rejiang@bnu.edu.cn

Huichun Zhang

Department of Mathematics, Sun Yat-sen University, Guangzhou 510275, China

E-mail address: zhanghc3@mail.sysu.edu.cn 